\title{
A note on partial calmness for bilevel optimization problems with linearly structured lower level
}

\author{
Patrick Mehlitz* Leonid I. Minchenko $^{\dagger} \quad$ Alain B. Zemkoho
}

June 30, 2020

\begin{abstract}
Partial calmness is a celebrated but restrictive property of bilevel optimization problems whose presence opens a way to the derivation of KarushKuhn-Tucker-type necessary optimality conditions in order to characterize local minimizers. In the past, sufficient conditions for the validity of partial calmness have been investigated. In this regard, the presence of a linearly structured lower level problem has turned out to be beneficial. However, the associated literature suffers from inaccurate results. In this note, we clarify some regarding erroneous statements and visualize the underlying issues with the aid of illustrative counterexamples.
\end{abstract}

Keywords: Bilevel optimization, Linear programming, Partial calmness

MSC: 49J53, 90C30

\section{Introduction}

We consider the standard bilevel optimization problem

$$
\min _{x, y}\{F(x, y) \mid x \in X, y \in S(x)\}
$$

\footnotetext{
${ }^{*}$ Brandenburgische Technische Universität Institute of Mathematics, 03046 Cottbus, Germany, mehlitz@b-tu.de, https://www.b-tu.de/fg-optimale-steuerung/team/dr-patrick-mehlitz, ORCID: 0000-00029355-850X

${ }^{\dagger}$ Belarus State University of Informatics and Radioelectronics, 6 P. Brovki Street, Minsk 220013, Belarus, leonidm@insoftgroup.com, ORCID: 0000-0002-8773-2559

${ }^{\ddagger}$ University of Southampton, School of Mathematics, Southampton SO17 1BJ, United Kingdom, a.b.zemkoho@soton.ac.uk, https://www.southampton.ac.uk/maths/about/staff/abz1e14.page, ORCID: 0000-0003-1265-4178
} 
where $F: \mathbb{R}^{n} \times \mathbb{R}^{m} \rightarrow \mathbb{R}$ is a locally Lipschitz continuous function, the set $X \subset \mathbb{R}^{n}$ is nonempty and closed, and $S: \mathbb{R}^{n} \rightrightarrows \mathbb{R}^{m}$ is the solution mapping of a standard parametric optimization problem, i.e.,

$$
\forall x \in \mathbb{R}^{n}: \quad S(x):=\underset{y}{\operatorname{argmin}}\{f(x, y) \mid g(x, y) \leq 0\} .
$$

Above, the data functions $f: \mathbb{R}^{n} \times \mathbb{R}^{m} \rightarrow \mathbb{R}$ and $g: \mathbb{R}^{n} \times \mathbb{R}^{m} \rightarrow \mathbb{R}^{q}$ are assumed to be continuous. The component functions of $g$ will be addressed by $g_{1}, \ldots, g_{q}: \mathbb{R}^{n} \times \mathbb{R}^{m} \rightarrow \mathbb{R}$. Nowadays, bilevel programming is one of the most intensively investigated topics in optimization theory since, on the one hand, there exist numerous underlying applications from economics, finance, chemistry, or engineering while, on the other hand, problems of this type are quite challenging, both theoretically and numerically, see Bard (1998); Dempe (2002); Dempe et al. (2015). In this note, we are concerned with the concept of partial calmness, fundamental in deriving necessary optimality conditions when the so-called optimal value function reformulation (a precise definition is stated below) is under consideration, see Section 3 for details.

To introduce this reformulation, we exploit the function $\varphi: \mathbb{R}^{n} \rightarrow \overline{\mathbb{R}}$ given by

$$
\forall x \in \mathbb{R}^{n}: \quad \varphi(x):=\inf _{y}\{f(x, y) \mid g(x, y) \leq 0\} .
$$

Clearly, $\varphi$ is the so-called optimal value function of the parametric optimization problem $\min _{y}\{f(x, y) \mid g(x, y) \leq 0\}$ which is referred to as the lower level problem of (BPP). It is well known that (BPP) is equivalent to its optimal value function reformulation defined by

$$
\min _{x, y}\{F(x, y) \mid x \in X, f(x, y)-\varphi(x) \leq 0, g(x, y) \leq 0\} .
$$

Observing that (OVR) is a single-level optimization problem, this reformulation approach opens a way to the theoretical and numerical treatment of (BPP). Due to the implicit character of $\varphi$, one has to observe that (OVR) is still a quite challenging problem since it is often not possible to compute a fully explicit representation of the function $\varphi$ in practice. Additionally, (OVR) is generally nonsmooth since $\varphi$ is nonsmooth in several practically relevant situations. Moreover, it is folklore that (OVR) is inherently irregular by definition of $\varphi$, i.e., standard constraint qualifications from (nonsmooth) optimization fail to hold at all feasible points of (OVR), see e.g. (Ye and Zhu, 1995, Proposition 3.2). Finally, let us mention that (OVR) is a nonconvex optimization problem in general even if all the data functions $F, f$, and $g_{1}, \ldots, g_{q}$ as well as the set $X$ are convex. Despite all these shortcomings, reformulating (BPP) via (OVR) became quite popular in the mathematical programming community. Starting with Outrata (1988), there appeared numerous publications which exploit (OVR) for the derivation of optimality conditions and solution algorithms for (BPP).

In the seminal paper Ye and Zhu (1995), the authors suggested to investigate the situation where $(x, y) \mapsto f(x, y)-\varphi(x)$ is a locally exact penalty function for (OVR) at some of its local minimizers $(\bar{x}, \bar{y}) \in \mathbb{R}^{n} \times \mathbb{R}^{m}$ in more detail. More precisely, they 
discussed conditions ensuring the existence of a finite scalar $\bar{\kappa}>0$ such that $(\bar{x}, \bar{y})$ is a local minimizer of the partially penalized problem

$$
\min _{x, y}\{F(x, y)+\kappa(f(x, y)-\varphi(x)) \mid x \in X, g(x, y) \leq 0\}
$$

for all $\kappa \geq \bar{\kappa}$, too. The authors called this property partial calmness of (BPP) at $(\bar{x}, \bar{y})$, see Definition 3.1 and Lemma 3.2, according to Clarke's classical notion of calmness for nonlinear optimization problems, see (Clarke, 1983, Section 6.4). Observing that $(\operatorname{OVR}(\kappa))$ may satisfy standard constraint qualifications, the presence of the partial calmness property opens a way to the derivation of necessary optimality conditions for (BPP) via (OVR). This has been done successfully in e.g. Dempe et al. (2007); Dempe and Franke (2015); Dempe and Zemkoho (2011, 2012, 2013); Mordukhovich et al. (2012); Ye and Zhu (1995, 2010). Recently, some Newton-type methods for the numerical solution of (BPP) have been developed which are based on the presence of the partial calmness property, see Fischer et al. (2019); Fliege et al. (2020). Let us note that the idea of partial calmness can be generalized to far more difficult settings, e.g. to bilevel optimal control problems or to situations where the lower level program is a parametric conic optimization problem, see e.g. Benita and Mehlitz (2016); Dempe et al. (2018); Mehlitz (2016); Ye (1995, 1997). Unfortunately, partial calmness is a quite restrictive property, see Henrion and Surowiec (2011), which only holds in very particular situations, e.g. where the lower level problem of (BPP) is fully linear, i.e., when the functions $f$ and $g$ are affine w.r.t. all variables, see (Ye and Zhu, 1995, Proposition 4.1). The latter result gave rise to a number of publications where the authors tried to generalize this observation to lower level problems where linearity is only present w.r.t. $y$, see e.g. Dempe and Zemkoho $(2012,2013)$. However, as we will see in this note, such a generalization is not possible in general. We present simple counterexamples which refute more general versions of (Ye and Zhu, 1995, Proposition 4.1). Furthermore, we point out the essential bug, originating from Ye and Zhu (1995), which caused the proof in e.g. (Dempe and Zemkoho, 2013, Theorem 4.2) to be erroneous.

The remaining parts of this note are organized as follows. In Section 2, we briefly summarize the notation and terminology used in this manuscript. We formally introduce the concept of partial calmness and recall some sufficient conditions guaranteeing its validity in Section 3. Section 4 is dedicated to the investigation of partial calmness in the context of bilevel optimization with linearly structured lower level problems. We first state a correct proof of the seminal result (Ye and Zhu, 1995, Proposition 4.1) which addresses fully linear lower level problems. Furthermore, we comment on the bug from the classical proof stated in Ye and Zhu (1995). By means of examples, we visualize that partial calmness does not need to be inherent as soon as the lower level problem is only linear w.r.t. the variable $y$. Reviewing some literature, we report on selected conditions which ensure validity of partial calmness in this situation. We finalize the paper by means of some concluding remarks in Section 5. 


\section{Notation}

In this manuscript, we mainly exploit standard notation. Without loss of generality, we equip all appearing spaces (including product structures) with the maximum norm $\|\cdot\|_{\infty}$. For some vector $x \in \mathbb{R}^{n}$ and a scalar $\varepsilon>0, \mathbb{U}_{\varepsilon}(x):=\left\{y \in \mathbb{R}^{n} \mid\|x-y\|_{\infty}<\varepsilon\right\}$ and $\mathbb{B}_{\varepsilon}(x):=\left\{y \in \mathbb{R}^{n} \mid\|x-y\|_{\infty} \leq \varepsilon\right\}$ denote the open and closed $\varepsilon$-ball around $x$, respectively. Furthermore, for an arbitrary set $A \subset \mathbb{R}^{n}$, we use

$$
\operatorname{dist}(x, A):=\inf \left\{\|x-y\|_{\infty} \mid y \in A\right\}
$$

in order to represent the distance of $x$ to $A$. For some matrix $M \in \mathbb{R}^{m \times n}$ and an index set $I \subset\{1, \ldots, m\}, M_{I} \in \mathbb{R}^{|I| \times n}$ is the matrix which results from $M$ by deleting all rows whose associated index does not belong to $I$. We use e $\in \mathbb{R}^{n}$ to denote the all-ones vector.

Let $\Upsilon: \mathbb{R}^{n} \rightrightarrows \mathbb{R}^{m}$ be a set-valued mapping. The domain and the graph of $\Upsilon$ are defined by $\operatorname{dom} \Upsilon:=\left\{x \in \mathbb{R}^{n} \mid \Upsilon(x) \neq \varnothing\right\}$ and gph $\Upsilon:=\left\{(x, y) \in \mathbb{R}^{n} \times \mathbb{R}^{m} \mid y \in \Upsilon(x)\right\}$, respectively. Recall that $\Upsilon$ is called inner semicontinuous at some point $(\bar{x}, \bar{y}) \in \operatorname{gph} \Upsilon$ whenever for each sequence $\left\{x_{k}\right\}_{k \in \mathbb{N}} \subset \mathbb{R}^{n}$ converging to $\bar{x}$, there is another sequence $\left\{y_{k}\right\}_{k \in \mathbb{N}} \subset \mathbb{R}^{m}$ converging to $\bar{y}$ such that $y_{k} \in \Upsilon\left(x_{k}\right)$ holds for all sufficiently large $k \in \mathbb{N}$. Assume that there are continuous functions $h_{1}, \ldots, h_{q}: \mathbb{R}^{n} \times \mathbb{R}^{m} \rightarrow \mathbb{R}$ such that $\Upsilon$ possesses the particular form

$$
\forall x \in \mathbb{R}^{n}: \quad \Upsilon(x):=\left\{y \in \mathbb{R}^{m} \mid h_{i}(x, y) \leq 0, i=1, \ldots, q\right\}
$$

and that $(\bar{x}, \bar{y}) \in \operatorname{gph} \Upsilon$ is chosen arbitrarily. Then $\Upsilon$ is called R-regular at $(\bar{x}, \bar{y})$ w.r.t. $\Omega \subset \mathbb{R}^{n}$ whenever there are constants $\kappa>0$ and $\varepsilon>0$ such that

$$
\begin{aligned}
& \forall(x, y) \in \mathbb{U}_{\varepsilon}(\bar{x}, \bar{y}) \cap\left(\Omega \times \mathbb{R}^{m}\right): \\
& \operatorname{dist}(y, \Upsilon(x)) \leq \kappa \max \left\{0, \max \left\{h_{i}(x, y) \mid i \in\{1, \ldots, q\}\right\}\right\}
\end{aligned}
$$

holds, see e.g. Bednarczuk et al. (2019). In case where this condition holds for $\Omega:=\mathbb{R}^{n}$, we simply say that $\Upsilon$ is R-regular at $(\bar{x}, \bar{y})$. Typically, one of the settings $\Omega:=\mathbb{R}^{n}$ or $\Omega:=\operatorname{dom} \Upsilon$ is under consideration. Due to (Bednarczuk et al., 2019, Theorem 5.1), Rregularity of $\Upsilon$ at some point of its graph is stronger than the so-called Aubin property, which is a prominent Lipschitzian property of set-valued mappings, provided that the functions $h_{1}, \ldots, h_{q}$ are locally Lipschitz continuous in a neighbourhood of the point of interest.

\section{Bilevel optimization and partial calmness}

Let us state the classical definition of partial calmness due to Ye and Zhu (1995). This property demands the problem (OVR) to behave stably in a certain sense w.r.t. small perturbations of the constraint $f(x, y)-\varphi(x) \leq 0$ at a given local minimizer of (BPP). Partial calmness originates from the classical notion of calmness for standard nonlinear optimization problems introduced in (Clarke, 1983, Section 6.4). 
Definition 3.1. Let $(\bar{x}, \bar{y}) \in \mathbb{R}^{n} \times \mathbb{R}^{m}$ be a local minimizer of (BPP). We say that (BPP) is partially calm at $(\bar{x}, \bar{y})$ if there exist $\delta>0$ and $\bar{\kappa}>0$ such that for each triplet $(x, y, u) \in \mathbb{B}_{\delta}(\bar{x}, \bar{y}, 0)$ satisfying

$$
x \in X, \quad f(x, y)-\varphi(x) \leq u, \quad g(x, y) \leq 0,
$$

we have $F(x, y)+\bar{\kappa}|u| \geq F(\bar{x}, \bar{y})$.

As we already mentioned in Section 1, it is clear from (Ye and Zhu, 1995, Proposition 3.3) that partial calmness of (BPP) at one of its local minimizers $(\bar{x}, \bar{y})$ is equivalent to $(x, y) \mapsto f(x, y)-\varphi(x)$ being a locally exact penalty function for (OVR) at $(\bar{x}, \bar{y})$. We summarize this observation in the subsequently stated lemma.

Lemma 3.2. Let $(\bar{x}, \bar{y}) \in \mathbb{R}^{n} \times \mathbb{R}^{m}$ be a local minimizer of (BPP). Then (BPP) is partially calm at $(\bar{x}, \bar{y})$ if and only if there is some $\bar{\kappa}>0$ such that $(\bar{x}, \bar{y})$ is a local minimizer of $(\operatorname{OVR}(\kappa))$ for each $\kappa \geq \bar{\kappa}$.

Next, we want to mention some criteria ensuring validity of the partial calmness condition. Therefore, we first introduce two set-valued mappings $\Gamma, \Phi: \mathbb{R}^{n} \rightrightarrows \mathbb{R}^{m}$ as stated below:

$$
\begin{aligned}
\forall x \in \mathbb{R}^{n}: \quad & \Gamma(x):=\left\{y \in \mathbb{R}^{m} \mid g(x, y) \leq 0\right\} \\
& \Phi(x):=\left\{y \in \mathbb{R}^{m} \mid f(x, y)-\varphi(x) \leq 0, g(x, y) \leq 0\right\} .
\end{aligned}
$$

Let us mention that gph $S$ and gph $\Phi$ actually coincide by definition of the optimal value function $\varphi$. However, $\Phi$ characterizes the lower level solution set with the aid of standard inequality constraints comprising the implicitly given function $\varphi$ while $S$ is completely implicit.

The following definitions are motivated by the considerations in the papers Bednarczuk et al. (2019); Henrion and Surowiec (2011); Ye and Zhu (1995), which, at least in parts, are concerned with bilevel optimization.

Definition 3.3. Fix some point $(\bar{x}, \bar{y}) \in \operatorname{gph} S$.

(i) We say that the lower level problem satisfies the local uniformly weak sharp minimum condition $(L U W S M C)$ at $(\bar{x}, \bar{y})$ whenever there are some $\alpha>0$ and $\varepsilon>0$ such that the following condition holds:

$$
\forall(x, y) \in \operatorname{gph} \Gamma \cap \mathbb{U}_{\varepsilon}(\bar{x}, \bar{y}): \quad \alpha \operatorname{dist}(y, S(x)) \leq f(x, y)-\varphi(x) .
$$

If the above condition can be strengthened to

$$
\forall(x, y) \in \operatorname{gph} \Gamma: \quad \alpha \operatorname{dist}(y, S(x)) \leq f(x, y)-\varphi(x),
$$

then the lower level problem is said to have a uniformly weak sharp minimum (UWSM). Particularly, the latter condition is independent of the point of interest $(\bar{x}, \bar{y})$. 
(ii) We say that the R-regularity constraint qualification (RRCQ) holds at $(\bar{x}, \bar{y})$ whenever $\Phi$ is $R$-regular at $(\bar{x}, \bar{y})$ w.r.t. $\operatorname{dom} \Phi$.

By definition, validity of UWSM implies that LUWSMC holds at all points in gph $S$. Both conditions originate from the notion of weak sharp minima which addresses constrained optimization problems, see Burke and Ferris (1993). Note that validity of RRCQ at some point $(\bar{x}, \bar{y}) \in \operatorname{gph} S$ implicitly demands that $\varphi$ is continuous around $\bar{x}$.

Below, we study the relationship between the conditions LUWSMC and RRCQ as well as their connection to the partial calmness property. First, we would like to show that whenever a given local minimizer of (BPP) satisfies LUWSMC, then (BPP) is partially calm at this point. This result generalizes related observations from (Ye and Zhu, 1995, Proposition 5.1) or (Henrion and Surowiec, 2011, Propositions 3.8 and 3.10).

Lemma 3.4. Let $(\bar{x}, \bar{y}) \in \mathbb{R}^{n} \times \mathbb{R}^{m}$ be a local minimizer of (BPP) where LUWSMC holds. Then (BPP) is partially calm at $(\bar{x}, \bar{y})$.

Proof. By local optimality of $(\bar{x}, \bar{y})$ for $(\mathrm{BPP})$, we find a constant $\gamma>0$ such that $F(x, y) \geq F(\bar{x}, \bar{y})$ holds for all $(x, y) \in \mathbb{B}_{\gamma}(\bar{x}, \bar{y})$ which are feasible to (BPP). Without loss of generality, we may assume that $F$ is Lipschitz continuous on $\mathbb{B}_{\gamma}(\bar{x}, \bar{y})$ with Lipschitz constant $L>0$. The assumptions of the lemma guarantee the existence of constants $\alpha>0$ and $\varepsilon>0$ such that (1) holds. Set $\delta:=\min \{\varepsilon / 2, \max \{\alpha, 1\} \gamma / 2\}$ and fix a triplet $(x, y, u) \in \mathbb{B}_{\delta}(\bar{x}, \bar{y}, 0)$ satisfying $x \in X, f(x, y)-\varphi(x) \leq u$, and $g(x, y) \leq 0$. Due to $(x, y) \in \operatorname{gph} \Gamma \cap \mathbb{U}_{\varepsilon}(\bar{x}, \bar{y})$, we find some point $\tilde{y} \in S(x)$ such that the estimate $\|y-\tilde{y}\|_{\infty} \leq(1 / \alpha)(f(x, y)-\varphi(x))$ holds. This yields

$$
\|\tilde{y}-\bar{y}\|_{\infty} \leq\|\tilde{y}-y\|_{\infty}+\|y-\bar{y}\|_{\infty} \leq(1 / \alpha) u+\|y-\bar{y}\|_{\infty} \leq \delta / \alpha+\delta \leq \gamma,
$$

and due to $x \in \mathbb{B}_{\gamma}(\bar{x})$, we find $(x, \tilde{y}) \in \mathbb{B}_{\gamma}(\bar{x}, \bar{y})$. Furthermore, this point is feasible to (BPP) which is why we obtain

$$
\begin{aligned}
F(x, y)-F(\bar{x}, \bar{y}) & \geq F(x, y)-F(x, \tilde{y}) \geq-L\|y-\tilde{y}\|_{\infty} \\
& \geq-(L / \alpha)(f(x, y)-\varphi(x)) \geq-(L / \alpha) u .
\end{aligned}
$$

Rearranging this inequality while noting that $(x, y, u) \in \mathbb{B}_{\delta}(\bar{x}, \bar{y}, 0)$ has been chosen arbitrarily, the lemma's assertion follows.

Next, we show that RRCQ is a sufficient condition for LUWSMC. This generalizes the recent result (Bednarczuk et al., 2019, Theorem 6.1).

Lemma 3.5. Let $(\bar{x}, \bar{y}) \in \operatorname{gph} S$ be chosen where $R R C Q$ holds. Furthermore, assume that there is some neighbourhood $U \subset \mathbb{R}^{n}$ of $\bar{x}$ such that $(\operatorname{dom} \Gamma) \cap U=(\operatorname{dom} S) \cap U$ is valid. Then LUWSMC holds at $(\bar{x}, \bar{y})$.

Proof. Noting that RRCQ holds at $(\bar{x}, \bar{y})$, the mapping $\Phi$ is R-regular at $(\bar{x}, \bar{y})$ w.r.t. $\operatorname{dom} \Phi$. Consequently, we find $\kappa>0$ and some $\varepsilon>0$ such that

$$
\forall(x, y) \in \mathbb{U}_{\varepsilon}(\bar{x}, \bar{y}) \cap\left(\operatorname{dom} \Phi \times \mathbb{R}^{m}\right):
$$




$$
\operatorname{dist}(y, \Phi(x)) \leq \kappa \max \left\{0, f(x, y)-\varphi(x), g_{1}(x, y), \ldots, g_{q}(x, y)\right\}
$$

holds. For each pair $(x, y) \in \mathbb{U}_{\varepsilon}(\bar{x}, \bar{y})$ with $g(x, y) \leq 0$, we automatically have the inequality $f(x, y) \geq \varphi(x)$ by definition of $\varphi$. Thus, we obtain

$$
\begin{aligned}
& \forall(x, y) \in \mathbb{U}_{\varepsilon}(\bar{x}, \bar{y}) \cap\left(\operatorname{dom} \Phi \times \mathbb{R}^{m}\right): \\
& \quad(x, y) \in \operatorname{gph} \Gamma \Longrightarrow \operatorname{dist}(y, \Phi(x)) \leq \kappa(f(x, y)-\varphi(x)) .
\end{aligned}
$$

Due to $\Phi(x)=S(x)$ for all $x \in \mathbb{R}^{n}$ and $(\operatorname{dom} \Phi) \cap U=(\operatorname{dom} \Gamma) \cap U$, this implies

$$
\begin{aligned}
& \forall(x, y) \in \mathbb{U}_{\varepsilon}(\bar{x}, \bar{y}) \cap\left(U \times \mathbb{R}^{m}\right): \\
& \quad(x, y) \in \operatorname{gph} \Gamma \Longrightarrow \operatorname{dist}(y, S(x)) \leq \kappa(f(x, y)-\varphi(x)) .
\end{aligned}
$$

Observing that we can find an open ball around $(\bar{x}, \bar{y})$ which is contained in the intersection $\mathbb{U}_{\varepsilon}(\bar{x}, \bar{y}) \cap\left(U \times \mathbb{R}^{m}\right)$, division by $\kappa$ shows that LUWSMC is valid at $(\bar{x}, \bar{y})$.

Note that the existence of a neighbourhood $U \subset \mathbb{R}^{n}$ of some given point $\bar{x} \in \operatorname{dom} \Gamma$ such that $(\operatorname{dom} \Gamma) \cap U=(\operatorname{dom} S) \cap U$ holds is not too restrictive. By continuity of $g$, we already know that $\Gamma$ possesses closed images. Thus, if $\Gamma$ is locally bounded around $\bar{x}$, the above condition is inherent by Weierstraß' theorem.

Recently, some conditions, which comprise a local constant rank assumption, implying validity of R-regularity have been derived in Bednarczuk et al. (2019). Applying this to the situation at hand, we obtain the following result.

Lemma 3.6. Fix some point $(\bar{x}, \bar{y}) \in \operatorname{gph} S$ where $S$ is inner semicontinuous. Furthermore, let the functions $f$ and $g_{1}, \ldots, g_{q}$ be locally Lipschitz continuous and continuously differentiable w.r.t. $y$ in a neighbourhood of $(\bar{x}, \bar{y})$. Let us denote the index set associated with all lower level inequality constraints which are active at $(\bar{x}, \bar{y})$ by $I(\bar{x}, \bar{y}):=\left\{i \in\{1, \ldots, q\} \mid g_{i}(\bar{x}, \bar{y})=0\right\}$. Setting $g_{0}(x, y):=f(x, y)-\varphi(x)$ for all $x \in \mathbb{R}^{n}$ and $y \in \mathbb{R}^{m}$, we assume that there is a neighbourhood $U \subset \mathbb{R}^{n} \times \mathbb{R}^{m}$ of $(\bar{x}, \bar{y})$ such that for each index set $J \subset I(\bar{x}, \bar{y}) \cup\{0\}$, the family $\left(\nabla_{y} g_{i}(x, y)\right)_{i \in J}$ has constant rank on $U$. Then $R R C Q$ is valid.

Proof. The assumptions of the lemma guarantee that for each index set $\tilde{J} \subset I(\bar{x}, \bar{y})$, the family $\left(\nabla_{y} g_{i}(x, y)\right)_{i \in \tilde{J}}$ possesses constant rank on $U$. Furthermore, $\Gamma$ is inner semicontinuous at $(\bar{x}, \bar{y})$ by inner semicontinuity of $S$ at this point. We now can apply (Bednarczuk et al., 2019, Theorem 4.2) in order to obtain that $\Gamma$ is R-regular at $(\bar{x}, \bar{y})$.

Observe that $\varphi$ is continuous at $\bar{x}$ since $S$ is inner semicontinuous at $(\bar{x}, \bar{y})$. Thus, we can invoke (Bednarczuk et al., 2019, Theorem 5.1) and (Mordukhovich and Nam, 2005, Theorem 5.2(i)) in order to see that $\varphi$ is already locally Lipschitz continuous at $\bar{x}$. Particularly, $\varphi$ is continuous in a neighbourhood of $\bar{x}$.

Thus, locally around $(\bar{x}, \bar{y})$, the variational description of $\Phi$ is provided by functions which are Lipschitz continuous and continuously differentiable w.r.t. $y$. By assumption, $\Phi$ is inner semicontinuous at $(\bar{x}, \bar{y})$ since $\Phi$ and $S$ actually coincide. Now, the desired result follows from (Bednarczuk et al., 2019, Theorem 4.2) again. 
Remark 3.7. Observe that the assumptions of Lemma 3.6 imply that the point of interest $(\bar{x}, \bar{y}) \in \mathbb{R}^{n} \times \mathbb{R}^{m}$ satisfies $\bar{x} \in \operatorname{int} \operatorname{dom} S$ since $S$ is supposed to be inner semicontinuous at $(\bar{x}, \bar{y})$. Thus, we have $\bar{x} \in \operatorname{int} \operatorname{dom} \Gamma$ as well. Consequently, the domains of $\Gamma$ and $S$ coincide locally around $\bar{x}$. Particularly, the assumptions of Lemma 3.6 already guarantee that LUWSMC holds at $(\bar{x}, \bar{y})$ due to Lemma 3.5.

In Figure 1, we depict the relations between the conditions from Definition 3.3 which are all sufficient for partial calmness at a given local minimizer of (BPP).

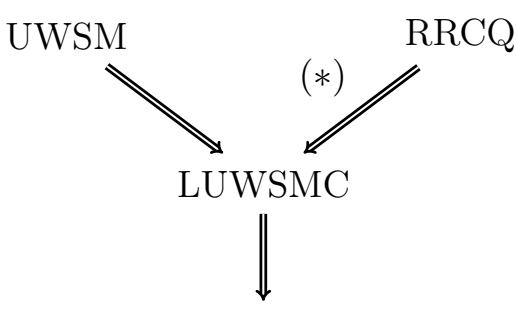

Partial calmness

Figure 1: Relations between conditions implying partial calmness at a given local minimizer $(\bar{x}, \bar{y})$ of $(\mathrm{BPP})$. Relation $(*)$ requires local coincidence of $\operatorname{dom} \Gamma$ and $\operatorname{dom} S$ around $\bar{x}$.

\section{Partial calmness and linear lower level problems}

In this section, we investigate the presence of partial calmness for the bilevel programming problem (BPP) where the lower level solution mapping $S$ is described by one of the following settings:

- the lower level problem is linear w.r.t. the decision variable $y$, i.e., there exist continuous functions $c: \mathbb{R}^{n} \rightarrow \mathbb{R}^{m}, A: \mathbb{R}^{n} \rightarrow \mathbb{R}^{q}$, and $B: \mathbb{R}^{n} \rightarrow \mathbb{R}^{q \times m}$ such that

$$
\forall x \in \mathbb{R}^{n}: \quad S(x):=\underset{y}{\operatorname{argmin}}\left\{c(x)^{\top} y \mid A(x)+B(x) y \leq 0\right\},
$$

- the lower level problem is a linear parametric optimization problem with continuous right-hand side perturbation, i.e., there exist matrices $c \in \mathbb{R}^{m}$ and $B \in \mathbb{R}^{q \times m}$ as well as a continuous function $A: \mathbb{R}^{n} \rightarrow \mathbb{R}^{q}$ such that

$$
\forall x \in \mathbb{R}^{n}: \quad S(x):=\underset{y}{\operatorname{argmin}}\left\{c^{\top} y \mid A(x)+B y \leq 0\right\},
$$

- the lower level problem is a linear parametric optimization problem with affine perturbations of the coefficients within the objective function, i.e., there exist matrices $c \in \mathbb{R}^{m}, C \in \mathbb{R}^{m \times n}, A \in \mathbb{R}^{q}$, and $B \in \mathbb{R}^{q \times m}$ such that

$$
\forall x \in \mathbb{R}^{n}: \quad S(x):=\underset{y}{\operatorname{argmin}}\left\{(C x+c)^{\top} y \mid A+B y \leq 0\right\} .
$$


Observe that the model (2) covers (3) and (4).

In (Ye and Zhu, 1995, Proposition 4.1), the authors show that bilevel programming problems with fully linear lower level problem (recall that this means that the functions $f$ and $g$ need to be affine, and this is covered by model (3) in case where $A$ is affine) are partially calm at all their local minimizers. As we will show below, this result is correct although the proof in Ye and Zhu (1995) comprises a small mistake. Subsequently, we present a slightly more general statement than the one from Ye and Zhu (1995), which addresses lower level problems of type (3), and state a corrected version of the proof. Afterwards, we point the reader's attention to the bug in Ye and Zhu (1995).

Theorem 4.1. Let $(\bar{x}, \bar{y}) \in \mathbb{R}^{n} \times \mathbb{R}^{m}$ be a local minimizer of (BPP) where the lower level solution mapping $S$ is given as in (3). Then (BPP) is partially calm at $(\bar{x}, \bar{y})$.

Proof. Choose $\delta>0$ arbitrarily and fix some point $(x, y, u) \in \mathbb{B}_{\delta}(\bar{x}, \bar{y}, 0)$ which satisfies

$$
x \in X, \quad c^{\top} y-\varphi(x) \leq u, \quad A(x)+B y \leq 0 .
$$

Noting that this implicitly demands $S(x) \neq \varnothing$, we can pick a vector

$$
y(x) \in \underset{z}{\operatorname{argmin}}\left\{\|y-z\|_{\infty} \mid z \in S(x)\right\}
$$

since $S(x)$ is a polyhedron and, thus, closed. As a consequence, we obtain

$$
\begin{aligned}
& \|y-y(x)\|_{\infty}=\min _{\sigma, z}\left\{\sigma \mid-\sigma \mathrm{e} \leq y-z \leq \sigma \mathrm{e}, c^{\top} z-\varphi(x) \leq 0, A(x)+B z \leq 0\right\} \\
& =\max _{\xi}\left\{\begin{array}{l|l}
y^{\top}\left(\xi_{1}-\xi_{2}\right)+\varphi(x) \xi_{3}-A(x)^{\top} \xi_{4} & \begin{array}{l}
\xi_{1}-\xi_{2}+c \xi_{3}+B^{\top} \xi_{4}=0, \\
-\mathrm{e}^{\top} \xi_{1}-\mathrm{e}^{\top} \xi_{2}=1, \\
\xi_{1}, \xi_{2}, \xi_{3}, \xi_{4} \leq 0
\end{array}
\end{array}\right\} \\
& \stackrel{(*)}{=} \max _{\xi}\left\{\begin{array}{l|l}
\left(\varphi(x)-c^{\top} y\right) \xi_{3}+(-A(x)-B y)^{\top} \xi_{4} & \begin{array}{l}
\xi_{1}-\xi_{2}+c \xi_{3}+B^{\top} \xi_{4}=0, \\
-\mathrm{e}^{\top} \xi_{1}-\mathrm{e}^{\top} \xi_{2}=1, \\
\xi_{1}, \xi_{2}, \xi_{3}, \xi_{4} \leq 0
\end{array}
\end{array}\right\}
\end{aligned}
$$

by strong duality of linear programming. Observing that the latter program possesses a solution, there exists a vertex $\left(\xi_{1}(x, y), \xi_{2}(x, y), \xi_{3}(x, y), \xi_{4}(x, y)\right)$ of the set

$$
Q:=\left\{\begin{array}{l|l}
\left(\xi_{1}, \xi_{2}, \xi_{3}, \xi_{4}\right) & \begin{array}{l}
\xi_{1}-\xi_{2}+c \xi_{3}+B^{\top} \xi_{4}=0, \\
-\mathrm{e}^{\top} \xi_{1}-\mathrm{e}^{\top} \xi_{2}=1, \\
\xi_{1}, \xi_{2}, \xi_{3}, \xi_{4} \leq 0
\end{array}
\end{array}\right\}
$$

which satisfies

$$
\begin{aligned}
\|y-y(x)\|_{\infty} & =\left(\varphi(x)-c^{\top} y\right) \xi_{3}(x, y)+(-A(x)-B y)^{\top} \xi_{4}(x, y) \\
& \leq\left(\varphi(x)-c^{\top} y\right) \xi_{3}(x, y) \leq|u|\left|\xi_{3}(x, y)\right| .
\end{aligned}
$$


Noting that the polyhedron $Q$ possesses only finitely many vertices and does not depend on the choice of $(x, y)$, there is some constant $M>0$ such that $\|y-y(x)\|_{\infty} \leq M|u|$ follows. Observing that $F$ is locally Lipschitz continuous, we find some constant $L>0$ such that $F$ is Lipschitz continuous with Lipschitz constant $L$ on the ball $\mathbb{B}_{(M+1) \delta}(\bar{x}, \bar{y})$ if only $\delta$ is small enough. Since we have

$$
\|y(x)-\bar{y}\|_{\infty} \leq\|y(x)-y\|_{\infty}+\|y-\bar{y}\|_{\infty} \leq M|u|+\|y-\bar{y}\|_{\infty} \leq(M+1) \delta
$$

and $\|x-\bar{x}\|_{\infty} \leq \delta$, we can choose $\delta$ so small such that $(x, y(x))$ always lies within the radius of local optimality associated with the local minimizer $(\bar{x}, \bar{y})$ of (BPP). Noting that $(x, y(x))$ is feasible to (BPP), it holds

$$
F(x, y)-F(\bar{x}, \bar{y}) \geq F(x, y)-F(x, y(x)) \geq-L\|y-y(x)\|_{\infty} \geq-L M|u| .
$$

Recalling that $(x, y, u) \in \mathbb{B}_{\delta}(\bar{x}, \bar{y}, 0)$ has been chosen arbitrarily, the statement of the theorem follows.

Let us recall that in (Ye and Zhu, 1995, Section 4.2), the authors consider the particular case where the function $A$ in the definition of (3) is affine.

Next, we specify where the bug in the original proof from Ye and Zhu (1995) is located. If not stated otherwise, the subsequently stated remarks address the lower level problem from (3).

Remark 4.2. In the classical proof of (Ye and Zhu, 1995, Proposition 4.1), it has been claimed that equality in $(*)$ holds with the right-hand side

$$
\max _{\xi}\left\{\begin{array}{l|l}
\left(\varphi(x)-c^{\top} y\right) \xi_{3}+(-A(x)-B y)^{\top} \xi_{4} & \begin{array}{l}
-\mathrm{e}^{\top} \xi_{1}-\mathrm{e}^{\top} \xi_{2}=1, \\
\xi_{1}, \xi_{2}, \xi_{3}, \xi_{4} \leq 0
\end{array}
\end{array}\right\}
$$

where the constraint $\xi_{1}-\xi_{2}+c \xi_{3}+B^{\top} \xi_{4}=0$ is deleted from the feasible set. Noting that this enlarges the feasible set of the program from the left-hand side of (*), the equality there needs to be replaced by the relation $\leq$. Even worse, it is obvious that whenever $y \notin S(x)$ holds, then, due to $\varphi(x)-c^{\top} y<0$, (5) possesses the optimal value $+\infty$. Thus, the authors obtained the trivial estimate $\|y-y(x)\|_{\infty} \leq+\infty$ in Ye and Zhu (1995) which is, for sure, of no use.

Clearly, the proof provided above cannot be generalized to the setting where $S$ is given as in (2), since in this case, the vertices of the set

$$
Q(x):=\left\{\begin{array}{l|l}
\left(\xi_{1}, \xi_{2}, \xi_{3}, \xi_{4}\right) & \begin{array}{l}
\xi_{1}-\xi_{2}+c(x) \xi_{3}+B(x)^{\top} \xi_{4}=0 \\
-\mathrm{e}^{\top} \xi_{1}-\mathrm{e}^{\top} \xi_{2}=1, \\
\xi_{1}, \xi_{2}, \xi_{3}, \xi_{4} \leq 0
\end{array}
\end{array}\right\}
$$

depend on the parameter $x$ which means that the existence of the constant $M$ in the proof of Theorem 4.1 does not come for free. These arguments also demonstrate that the proof of (Dempe and Zemkoho, 2013, Theorem 4.2) is not correct since it reprises the original bug from Ye and Zhu (1995). 
Remark 4.3. Inspecting the proof of Theorem 4.1 carefully, one can observe that the condition

$$
\begin{aligned}
\exists M>0 \quad \forall(x, y) & \in \mathbb{R}^{n} \times \mathbb{R}^{m}: \\
A(x)+B y & \leq 0 \Longrightarrow \operatorname{dist}(y, S(x)) \leq M\left(c^{\top} y-\varphi(x)\right)
\end{aligned}
$$

has been verified, and the latter already means that the parametric lower level optimization problem associated with (3) possesses a UWSM, see Definition 3.3. Recently, this has been pointed out in (Minchenko and Berezhnov, 2017, Lemma 2.1).

Remark 4.4. Consider the situation where the mapping $A$ is affine. In this case, it is well known that the solution map $S$ associated with (3) is inner semicontinuous at each point $(\bar{x}, \bar{y}) \in \operatorname{gph} S$ which satisfies $\bar{x} \in \operatorname{int} \operatorname{dom} S$. Thus, Lemma 3.6 guarantees that at any such point, $R R C Q$ is valid since the remaining constant rank assumption trivially holds observing that all appearing gradients w.r.t. $y$ are constant.

In the light of Remark 4.2, one now might ask whether the result of Theorem 4.1 can be generalized to the setting where the lower level problem is given as in (2) as proposed in (Dempe and Zemkoho, 2013, Theorem 4.2). As the following example shows, this is, unluckily, not the case.

Example 4.5. Let us consider the bilevel optimization problem

$$
\min _{x, y}\{-x+y \mid x \leq 2, y \in S(x)\}
$$

where $S: \mathbb{R} \rightrightarrows \mathbb{R}$ is given by

$$
\forall x \in \mathbb{R}: \quad S(x):=\underset{y}{\operatorname{argmin}}\left\{-x^{2} y \mid y \in[0,1]\right\} .
$$

One can easily check that

$$
\forall x \in \mathbb{R}: \quad S(x):=\left\{\begin{array}{ll}
\{1\} & \text { if } x \neq 0, \\
{[0,1]} & \text { if } x=0
\end{array} \quad \text { and } \quad \varphi(x):=-x^{2}\right.
$$

hold true, i.e., $(\bar{x}, \bar{y}):=(0,0)$ is a local minimizer of the given bilevel programming problem. Furthermore, the global minimizer of this bilevel optimization problem is given by $(\tilde{x}, \tilde{y}):=(2,1)$.

Now, for $\kappa>0$, let us consider the associated partially penalized problem $(\mathrm{OVR}(\kappa))$ which reads as

$$
\min _{x, y}\left\{-x+y+\kappa x^{2}(1-y) \mid x \leq 2, y \in[0,1]\right\} .
$$

The sequence $\left\{\left(\frac{1}{k}, 0\right)\right\}_{k \in \mathbb{N}}$ is feasible for the latter and converges to $(\bar{x}, \bar{y})$. The associated objective values of (6) are given by $\left\{-\frac{1}{k}+\kappa \frac{1}{k^{2}}\right\}_{k \in \mathbb{N}}$. Observe that for sufficiently large $k \in \mathbb{N}$, the elements of this sequence are negative. Particularly, there is no finite $\kappa>0$ such that $(\bar{x}, \bar{y})$ is a local minimizer of (6). Due to Lemma 3.2, the bilevel optimization problem under consideration cannot be partially calm at $(\bar{x}, \bar{y})$. 
The above example refutes (Dempe and Zemkoho, 2013, Theorem 4.2). The proof provided in the latter paper essentially adapted the one from Ye and Zhu (1995) and the bug therein, see Remark 4.2. In contrast to Ye and Zhu (1995), where this mistake did not effect the correctness of the result, the statement from Dempe and Zemkoho (2013) is not true in general.

In the light of Lemma 3.4, the following result, however, provides a sufficient condition for partial calmness in cases where the lower level problem is given as in (2). It follows directly from Lemma 3.6, see Remark 3.7 as well.

Theorem 4.6. Let us assume that the solution map $S: \mathbb{R}^{n} \rightrightarrows \mathbb{R}^{m}$ is given as in (2) where the matrix functions $c, A$, and $B$ are assumed to be locally Lipschitz continuous. Fix a point $(\bar{x}, \bar{y}) \in \operatorname{gph} S$ where $S$ is inner semicontinuous. Furthermore, set

$$
\forall x \in \mathbb{R}^{n}: \quad \mathcal{B}(x):=\left[\begin{array}{c}
c(x)^{\top} \\
B(x)_{I(\bar{x}, \bar{y})}
\end{array}\right]
$$

and assume that there is a neighbourhood $U \subset \mathbb{R}^{n}$ of $\bar{x}$ such that for each index set $J \subset\{1, \ldots,|I(\bar{x}, \bar{y})|+1\}$, the matrix $\mathcal{B}(x)_{J}$ has constant row rank for all $x \in U$. Then $R R C Q$ holds at $(\bar{x}, \bar{y})$.

Observe that due to Remark 4.4, Theorem 4.6 covers Theorem 4.1 in the setting where $A$ is an affine function while $c$ and $B$ are constant. Clearly, the assumptions of Theorem 4.6 cannot be neglected when considering partial calmness of (BPP) - checking Example 4.5, one obtains that both the inner semicontinuity assumption on $S$ and the constant rank assumption are violated at the point of interest. Below, we present an example where all these assumptions hold.

Example 4.7. We consider the lower level solution map $S: \mathbb{R} \rightrightarrows \mathbb{R}$ given in Example 4.5 at $(\tilde{x}, \tilde{y}):=(2,1)$. Clearly, $S$ is inner semicontinuous at $(\tilde{x}, \tilde{y})$ and the matrix

$$
\forall x \in \mathbb{R}: \quad \mathcal{B}(x)=\left[\begin{array}{c}
-x^{2} \\
1
\end{array}\right]
$$

satisfies the constant rank assumption from Theorem 4.6 in a neighbourhood of $\tilde{x}$. Thus, $R R C Q$ is valid at $(\tilde{x}, \tilde{y})$. In the light of Remark 3.7 and Lemma 3.4, the bilevel optimization problem from Example 4.5 is partially calm at its global minimizer $(\tilde{x}, \tilde{y})$.

The next example illustrates that even in the presence of inner semicontinuity of the solution mapping $S$ at the point of interest, partial calmness does not need to be inherent for problems with the lower level problem (2) if the constant rank assumption from Theorem 4.6 is violated.

Example 4.8. Let us investigate the bilevel optimization problem

$$
\min _{x, y}\left\{x y_{1} \mid y \in S(x)\right\}
$$

where $S: \mathbb{R} \rightrightarrows \mathbb{R}^{2}$ is given by

$$
\forall x \in \mathbb{R}: \quad S(x):=\underset{y}{\operatorname{argmin}}\left\{-x^{2} y_{2} \mid y_{2} \leq 0,-x y_{1}+y_{2} \leq 0\right\} .
$$


One can easily check that the corresponding solution mapping $S$ and the optimal value function $\varphi$, respectively, take the following precise forms:

$$
\forall x \in \mathbb{R}: \quad S(x)=\left\{\begin{array}{ll}
(-\infty, 0] \times\{0\} & x<0, \\
\mathbb{R} \times(-\infty, 0] & x=0, \\
{[0, \infty) \times\{0\}} & x>0
\end{array} \quad \text { and } \quad \varphi(x)=0 .\right.
$$

Furthermore, one can check that $(\bar{x}, \bar{y}):=(0,(0,0))$ is a global minimizer of the given bilevel optimization problem. Clearly, $S$ is inner semicontinuous at this point. The associated matrix $\mathcal{B}$ given by

$$
\forall x \in \mathbb{R}: \quad \mathcal{B}(x)=\left[\begin{array}{cc}
0 & -x^{2} \\
0 & 1 \\
-x & 1
\end{array}\right]
$$

does not satisfy the constant rank assumption at $\bar{x}$.

For $\kappa>0$, we consider the associated partially penalized problem $(\mathrm{OVR}(\kappa))$ given by

$$
\min _{x, y}\left\{x y_{1}-\kappa x^{2} y_{2} \mid y_{2} \leq 0,-x y_{1}+y_{2} \leq 0\right\} .
$$

Investigating the feasible sequence $\left\{\left(\frac{1}{k},\left(-\frac{1}{k},-\frac{1}{k^{2}}\right)\right)\right\}_{k \in \mathbb{N}}$, the associated objective values are $\left\{\frac{\kappa}{k^{4}}-\frac{1}{k^{2}}\right\}_{k \in \mathbb{N}}$, and this shows that $(\bar{x}, \bar{y})$ is not a local minimizer of the latter problem for any $\kappa>0$ since the elements of the latter sequence are negative for sufficiently large $k \in \mathbb{N}$. Hence, the underlying bilevel optimization problem is not partially calm at $(\bar{x}, \bar{y})$.

Let us briefly mention that (Ye, 1998, Theorem 3.4) provides a condition which ensures that the lower level problem (2) even possesses a UWSM, see Definition 3.3. On the other hand, in (Henrion and Surowiec, 2011, Example 3.9), it has been shown that already parametric optimization problems of type (4) do not necessarily satisfy LUWSMC and, thus, cannot possess a UWSM, i.e., the assumptions of (Ye, 1998, Theorem 3.4) are not generally satisfied for this class of lower level problems. In the light of Remark 4.3, this observation already underlines that trying to adapt the proof of Theorem 4.1 is hopeless in order to infer the partial calmness condition for bilevel programming problems with lower level problem (4) and, thus, (2). Furthermore, we would like to note that the solution mapping from (4) is not generally inner semicontinuous at the points of its graph which restricts the applicability of Theorem 4.6.

We want to close this section with an example which illustrates that bilevel optimization problems with lower level problems of type (4) indeed do not need to be partially calm at their respective local minimizers. This underlines that standard models from bilevel road pricing as discussed in Dempe and Franke (2015); Dempe and Zemkoho (2012) are not generally partially calm at their local minimizers without additional assumptions.

Example 4.9. We investigate the bilevel optimization problem

$$
\min _{x, y}\left\{x_{2}(y+1) \mid x_{1}=x_{2}^{2}, y \in S(x)\right\}
$$


where $S: \mathbb{R}^{2} \rightrightarrows \mathbb{R}$ is given by

$$
\forall x \in \mathbb{R}^{2}: \quad S(x):=\underset{y}{\operatorname{argmin}}\left\{x_{1} y \mid y \in[-1,1]\right\} .
$$

One obtains

$$
\forall x \in \mathbb{R}^{2}: \quad S(x)=\left\{\begin{array}{ll}
\{-1\} & x_{1}>0, \\
{[-1,1]} & x_{1}=0, \\
\{1\} & x_{1}<0
\end{array} \quad \text { and } \quad \varphi(x)=-\left|x_{1}\right|\right.
$$

by simple calculations. One can easily check that each feasible point of this bilevel optimization problem possesses objective value 0 . Particularly, $(\bar{x}, \bar{y}):=((0,0),-1)$ is one of its global minimizers. Next, for arbitrary $\kappa>0$, we consider the associated partially perturbed problem $(\mathrm{OVR}(\kappa))$ given by

$$
\min _{x, y}\left\{x_{2}(y+1)+\kappa\left(x_{1} y+\left|x_{1}\right|\right) \mid x_{1}=x_{2}^{2}, y \in[-1,1]\right\} .
$$

We investigate the feasible sequence $\left\{\left(\left(\frac{1}{k^{2}},-\frac{1}{k}\right),-1+\frac{1}{k}\right)\right\}_{k \in \mathbb{N}}$ which converges to $(\bar{x}, \bar{y})$. The associated sequence of objective values is given by $\left\{\frac{\kappa}{k^{3}}-\frac{1}{k^{2}}\right\}_{k \in \mathbb{N}}$, and the latter is negative for sufficiently large $k \in \mathbb{N}$. This shows that $(\bar{x}, \bar{y})$ is not a local minimizer of (7), and due to Lemma 3.2, the bilevel programming problem under consideration cannot be partially calm at $(\bar{x}, \bar{y})$.

We would like to point the reader's attention to the fact that the upper level feasible set $X$ in Example 4.9 is not a polyhedron, which is the striking idea behind the construction of this counterexample. It is, however, an open question whether or not bilevel optimization problems with lower level problem (4) and polyhedral $X$ are partially calm at their respective local minimizers. Observing that the solution mapping $S$ is a polyhedral set-valued mapping in this case (i.e., its graph can be represented as the union of finitely many convex polyhedral sets), this indeed might be possible since the associated feasible set of the bilevel optimization problem (BPP) is the union of finitely many convex polyhedral sets, and problems of this type are likely to be calm in Clarke's sense due to (Robinson, 1981, Proposition 1).

\section{Conclusions}

This manuscript underlines the well known fact that the property of a bilevel optimization problem to be partially calm at one of its local minimizers is quite restrictive. With the aid of three simple counterexamples, we have shown that this observation already addresses situations where the lower level problem is linear w.r.t. the lower level decision maker's variable. Our respective analysis refutes the result (Dempe and Zemkoho, 2013, Theorem 4.2). On the way, we revealed and corrected a bug in the proof of the seminal result (Ye and Zhu, 1995, Proposition 4.1) which has been spread over the literature about bilevel optimization. 


\section{References}

J. F. Bard. Practical Bilevel Optimization: Algorithms and Applications. Kluwer Academic, Dordrecht, 1998.

E. M. Bednarczuk, L. I. Minchenko, and K. E. Rutkowski. On Lipschitz-like continuity of a class of set-valued mappings. Optimization, pages 1-15, 2019. doi: 10.1080/02331934.2019.1696339.

F. Benita and P. Mehlitz. Bilevel Optimal Control With Final-State-Dependent FiniteDimensional Lower Level. SIAM Journal on Optimization, 26(1):718-752, 2016. doi: 10.1137/15M1015984.

J. V. Burke and M. C. Ferris. Weak Sharp Minima in Mathematical Programming. SIAM Journal on Control and Optimization, 31(5):1340-1359, 1993. doi: 10.1137/0331063.

F. H. Clarke. Optimization and Nonsmooth Analysis. Wiley, New York, 1983.

S. Dempe. Foundations of Bilevel Programming. Kluwer, Dordrecht, 2002.

S. Dempe and S. Franke. The bilevel road pricing problem. International Journal of Computing and Optimization, 2(2):71-92, 2015. doi: 10.12988/ijco.2015.5415.

S. Dempe and A. B. Zemkoho. The Generalized Mangasarian-Fromovitz Constraint Qualification and Optimality Conditions for Bilevel Programs. Journal of Optimization Theory and Applications, 148(1):46-68, 2011. doi: 10.1007/s10957-010-9744-8.

S. Dempe and A. B. Zemkoho. Bilevel road pricing: theoretical analysis and optimality conditions. Annals of Operations Research, 196(1):223-240, 2012. doi: 10.1007/s10479-011-1023-z.

S. Dempe and A. B. Zemkoho. The bilevel programming problem: reformulations, constraint qualifications and optimality conditions. Mathematical Programming, 138(1): 447-473, 2013. doi: 10.1007/s10107-011-0508-5.

S. Dempe, J. Dutta, and B. S. Mordukhovich. New necessary optimality conditions in optimistic bilevel programming. Optimization, 56(5-6):577-604, 2007. doi: 10.1080/02331930701617551.

S. Dempe, V. Kalashnikov, G. Pérez-Valdéz, and N. Kalashnykova. Bilevel Programming Problems - Theory, Algorithms and Applications to Energy Networks. Springer, Berlin, 2015.

S. Dempe, F. Mefo Kue, and P. Mehlitz. Optimality Conditions for Special Semidefinite Bilevel Optimization Problems. SIAM Journal on Optimization, 28(2):1564-1587, 2018. doi: 10.1137/16M1099303. 
A. Fischer, A. B. Zemkoho, and S. Zhou. Semismooth Newton-type method for bilevel optimization: Global convergence and extensive numerical experiments. preprint arXiv, pages 1-27, 2019. URL https://arxiv.org/abs/1912.07079.

J. Fliege, A. Tin, and A. B. Zemkoho. Gauss-Newton-type methods for bilevel optimization. preprint arXiv, pages 1-22, 2020. URL https://arxiv.org/abs/2003.03128.

R. Henrion and T. Surowiec. On calmness conditions in convex bilevel programming. Applicable Analysis, 90(6):951-970, 2011. doi: 10.1080/00036811.2010.495339.

P. Mehlitz. Bilevel programming problems with simple convex lower level. Optimization, 65(6):1203-1227, 2016. doi: 10.1080/02331934.2015.1122006.

L. I. Minchenko and D. E. Berezhnov. On global partial calmness for bilevel programming problems with linear lower-level problem. In CEUR Workshop Proceedings, volume 1987, 2017. URL http://ceur-ws.org/Vol-1987/paper60.pdf.

B. S. Mordukhovich and N. M. Nam. Variational Stability and Marginal Functions via Generalized Differentiation. Mathematics of Operations Research, 30(4):800-816, 2005. doi: $10.1287 /$ moor.1050.0147.

B. S. Mordukhovich, N. M. Nam, and H. M. Phan. Variational Analysis of Marginal Functions with Applications to Bilevel Programming. Journal of Optimization Theory and Applications, 152(3):557-586, 2012. doi: 10.1007/s10957-011-9940-1.

J.. V. Outrata. A note on the usage of nondifferentiable exact penalties in some special optimization problems. Kybernetika, pages 251-258, 1988 . URL http://eudml.org/doc/27574.

S. M. Robinson. Some continuity properties of polyhedral multifunctions. In H. König, B. Korte, and K. Ritter, editors, Mathematical Programming at Oberwolfach, pages 206-214. Springer, Berlin, 1981. doi: 10.1007/BFb0120929.

J. J. Ye. Necessary Conditions for Bilevel Dynamic Optimization Problems. SIAM Journal on Control and Optimization, 33(4):1208-1223, $1995 . \quad$ doi: $10.1137 /$ S0363012993249717.

J. J. Ye. Optimal Strategies For Bilevel Dynamic Problems. SIAM Journal on Control and Optimization, 35(2):512-531, 1997. doi: 10.1137/S0363012993256150.

J. J. Ye. New Uniform Parametric Error Bounds. Journal of Optimization Theory and Applications, 98(1):197-219, 1998. doi: 10.1023/A:1022649217032.

J. J. Ye and D. Zhu. New Necessary Optimality Conditions for Bilevel Programs by Combining the MPEC and Value Function Approaches. SIAM Journal on Optimization, 20(4):1885-1905, 2010. doi: 10.1137/080725088.

J. J. Ye and D. L. Zhu. Optimality conditions for bilevel programming problems. Optimization, 33(1):9-27, 1995. doi: 10.1080/02331939508844060. 